\title{
8 Sprachgebrauch und Interaktion
}

Die dritte Fallstudie zu Visualisierungen sprachlicher Daten ist von etwas anderem Charakter als die beiden anderen Studien. Es geht dabei um eine kritische Reflexion zur zentralen diagrammatischen Form der interaktionalen Linguistik bzw. der Gesprächslinguistik, dem Transkript. Ich habe an mehreren Stellen bereits auf die Bedeutung der Transkriptionstechnik für die Analyse gesprochener Sprache hingewiesen (vgl. Abschnitte 3.1 und 5.3). Die diagrammatische Grundfigur der Partitur ist dabei ein entscheidender Gedanke, um die Simultaneität von Stimmen sichtbar und damit analysierbar zu machen. Auch wenn nicht alle Transkriptionsstandards explizit von Partiturschreibweise o.ä. sprechen, finden sich doch immer Auszeichnungsmethoden, um zumindest bei Überlappungen Simultaneität von Gesprächsbeiträgen darzustellen.

Ich möchte in den folgenden Abschnitten nun die Frage diskutieren, ob Gesprächstranskripte überhaupt (noch) die richtige Form sind, gesprochene Sprache analysierbar zu machen, insbesondere vor dem Hintergrund neuerer theoretischer Überlegungen in der interaktionalen Linguistik und der Gesprächslinguistik. Zudem stellt sich die Frage, wie gesprächsanalytische Fragestellungen durch eine korpuslinguistische Perspektive inspiriert werden könnte und welche Konsequenzen dies auf die diagrammatische Darstellung zeitigt.

Ich stelle dafür zunächst wichtige Grundüberlegungen zu theoretischen Prämissen der interaktionalen Linguistik bzw. der Gesprächslinguistik an, um dann darauf basierend neue Visualisierungsformen vorzuschlagen.

\subsection{Grundüberlegungen zur Analyse von Gesprächen}

Mit einer Transkription von gesprochener Sprache, die Simultaneität sowie verbale, paraverbale und nonverbale Signale sorgfältig verschriftlicht und somit entzeitlicht, ist eine wichtige Grundlage für die Analyse geschaffen. Eine Grundlage, mit der es möglich wird, gesprochene Sprache nicht zu den Bedingungen und damit mit dem Maßstab geschriebener Sprache zu analysieren, sondern als eigene Modalität. Die Transkription ist damit auch eine Grundlage, um Sprachhandeln aus ethnomethodologischer, pragmatischer Sicht zu untersuchen. Diese Perspektive ist dabei davon geprägt, Alltagshandeln im Detail, empirisch genau zu beobachten und

als selbstverständlich hingenommene Praktiken und Verfahren [...] zu bestimmen, mittels derer die Mitglieder einer Gesellschaft [...] in ihrem Handeln das eigene Tun wahrnehmbar 
und erkennbar machen und die Wirklichkeit um sich sinnhaft strukturieren und ordnen. (Bergmann 2010, 51)

Vor diesem Hintergrund ist klar, dass eine möglichst detaillierte Transkription und eine detaillierte Analyse von kurzen Sequenzen zentral ist, um damit zu einem umfassenderen Verständnis zu gelangen, wie diese zu einer abstrakteren Makroperspektive führen, also gemeinschaftliches Handeln und gemeinschaftliche Organisation modellieren.

Als Korpuslinguistin oder Korpuslinguist ist man manchmal ob dieser Perspektive verwundert: Die Ziele sind ähnlich, vor allem bei einer sozial- und kulturwissenschaftlich interessierten Korpuslinguistik (Bubenhofer 2009; Felder et al. 2011; Scharloth/Bubenhofer 2011), die Methodologie unterscheidet sich jedoch deutlich, da die Indizien für kommunikatives und gemeinschaftlich sinnstiftendes Handeln eher in musterhaftem Sprachgebrauch im weitesten Sinne gesehen werden, also in einem Phänomen, das bei einer Analyse aus quantitativer Sicht zutage tritt, wie die beiden Studien zu den Geokollokationen (Kapitel 6) und Narrativen (Kapitel 7) zeigten. Allerdings muss auch gesagt werden, dass die Korpuslinguistik generell, auch die oben erwähnte Richtung, relativ blind gegenüber gesprochener Sprache ist, was zu Teilen damit erklärt werden kann, dass erst jüngst genügend große Mengen gesprochener Sprache als Korpora aufbereitet zur Verfügung stehen. ${ }^{95}$ Doch davon abgesehen verwundern die methodologischen Differenzen doch. Darauf werde ich später noch zu sprechen kommen.

Der Pragmatikbegriff ist für die Gesprächslinguistik ${ }^{96}$ und die Korpuspragmatik, also die sozial- und kulturlinguistisch interessierte Korpuslinguistik zentral. Arnulf Deppermann, der wichtige interaktionslinguistische Studien durchführte, stellt in „Pragmatik revisited“ aber fest, dass die empirische Forschung eine Neuakzentuierung des Begriffs nahelegt:

Ich plädiere dafür, vier Bestimmungsstücke ins Zentrum der Auffassung von „Pragmatik“ zu rücken, die traditionell nicht als zentrale Aspekte von „Pragmatik“ gesehen wurden: Leiblichkeit, Zeitlichkeit, Sozialität und Epistemizität. Zusammengenommen führen sie zu einem Verständnis von Pragmatik als der Wissenschaft vom sprachlich-leiblichen Handeln von soziohistorischen Subjekten in Raum und Zeit. (Deppermann 2015, 327)

95 Dazu zählt insbesondere die Datenbank für Gesprochenes Deutsch (DGD) am Institut für Deutsche Sprache (Mannheim), https://dgd.ids-mannheim.de/ (letzter Zugriff: 22. 9. 2020), vgl. Schmidt (2014).

96 Wenn ich von „Gesprächslinguistik“ ohne weiteren Präzisierungen rede, meine ich damit Gesprächslinguistik / Konversationsanalyse und interaktionale Linguistik insgesamt. 
Deppermann plausibilisiert alle vier Aspekte mit gesprächsanalytischen Beispielen. Für meine weitere Argumentation sind insbesondere Zeitlichkeit und Sozialität entscheidend, da ich bei diesen Potenzial für weiterführende diagrammatische Überlegungen sehe, doch ich möchte alle vier kurz skizzieren.

Unter „Leiblichkeit“ versteht Deppermann die intrinsische Verwobenheit von Sprache mit „Sichtbarem und Tastbarem, mit Objekten und Räumen, mit Positionen und Bewegungen, mit Aufmerksamkeit und Wahrnehmung“, die „Pragmatik muss deshalb Abschied nehmen vom Modell ,Sprecher-Hörer' als kommunikativer Grundkonstellation“ (Deppermann 2015, 330). Dies bedeutet, dass sprachliches Handeln als multimodale Koordination im Raum-Zeit-Kontinuum aufgefasst und analysiert werden muss.

Es ist augenscheinlich, dass ein Gesprächstranskript nicht in der Lage ist, Daten gesprochener Sprache so zu visualisieren, dass die Komplexität der kommunikativen Situation so umfassend analysierbar ist. Naheliegend ist es, neben dem Gesprächstranskript weitere Visualisierungen wie Videoaufnahme, Situationsskizzen und dergleichen einzusetzen (vgl. dazu Norris 2004), aber auch zusätzlich andere Datentypen zu erheben, wie etwa Blickrichtungen über Eye Tracking. Solche verschiedenartige Datentypen diagrammatisch $\mathrm{zu}$ integrieren ist eine besondere Herausforderung, da verschiedene diagrammatische Grundtypen und Organisationsprinzipien zusammengeführt werden müssen, insbesondere Sequenzialität, dreidimensionaler Raum und Pfade im Raum.

„Zeitlichkeit“ ist ein weiterer bedeutender Aspekt interaktionaler Kommunikation:

Es geht [der Pragmatik, NB] zum einen darum, grundlegende Mechanismen der zeitlichen Strukturierung des Handelns zu erfassen, zum anderen pragmatische Phänomene selbst in ihrer zeitlichen Struktur zu rekonstruieren (Deppermann 2015, 331).

Dabei geht es nicht bloß um Sequenzialität auf Mikroebene (turn-Abfolgen), sondern um weitergehende Bezüge auf Vergangenheit, Gegenwart und Zukunft im Verlaufe einer Interaktion, die Beiträge zur Handlungsbedeutung eines turns liefern (Deppermann 2015, 333).

Auch eine Zeitlichkeit mit solchen weitergehenden Bezügen wird in einem Gesprächstranskript nur ungenügend repräsentiert. Seine (gewollte) sequenzielle und polyphone Ausrichtung als (angestrebte) 1:1-Repräsentation des Gesprächsflusses ist hier hinderlich, da Bezüge in die Vergangenheit und die Zukunft auch an diese Linearität gebunden sind.

Der dritte Aspekt, „Sozialität“, umfasst wiederum zwei Komponenten. Einerseits geht es um die soziale Konstitution von Handlungsbedeutungen (Deppermann 2015, 335ff.). Diese müssen als Prozessphänomen aufgefasst werden, in 
dessen Verlauf durch gegenseitige Verstehensdokumentation gemeinschaftlich eine Handlungsbedeutung erarbeitet wird, etwa durch Nachfragen, Paraphrasieren und wiederum den Reaktionen darauf. Wenn die soziale Konstitution des Handels vernachlässigt wird, werden wichtige Dimensionen pragmatischer Bedeutung ausgeblendet (Deppermann 2015, 337): So etwa in kommunikativen Gattungen sedimentierte sozialtypisch geregelte Interaktionszusammenhänge und Erwartungsstrukturen oder die sozialsymbolische Kraft des Handelns, mit der sowohl Situationen (z. B. eine Vorlesung) als auch das dafür nötige Personal konstituiert wird.

Die zweite Komponente von „Sozialität“ fokussiert die Beteiligungsstrukturen, insbesondere in Mehrpersonenkonstellationen, wo „die Beteiligten vor Aufgaben der pragmatischen Organisation [stehen], die im Sprecher-Hörer-Modell nicht zu beschreiben sind“ (Deppermann 2015, 338). So gibt es in solchen Konstellationen verschiedene Rollen, wie „side participants (unadressierter Teilnehmer), overhearer (nicht beteiligter Zuhörer), eavesdropper (Lauscher), indirect target (nicht-adressierter Teilnehmer, für den eine Botschaft nicht bestimmt ist) usw.“ (Deppermann 2015, 338). Oder es spielen funktionsrollenbezogenes Wissen und Routinewissen über Handlungsabläufe als soziale Handlungserwartungen und Wissensvoraussetzungen eine tragende Rolle, um die entstehende Handlungsbedeutung rekonstruieren zu können.

Wenn „Sozialität“ bei einer Gesprächsanalyse gebührend beachtet werden soll, stößt auch hier das traditionelle Gesprächstranskript an Grenzen, da beispielsweise nur schon die Rollen der Teilnehmenden bezüglich ihrer Beteiligungsart in einer Mehrpersonenkonstellation in traditionellen Gesprächsvisualisierungen nicht einfach so auf einen Blick ersichtlich sind, da der Überblick (z. B. alleine zur Anzahl der turns) fehlt.

Schließlich gilt es, „Epistemizität“ in sozialen Interaktionen zu berücksichtigen. Damit sind die Wissensbestände (common ground) selbst gemeint, die zur Gestaltung sprachlichen Handelns nötig sind. Dabei muss aber die Prozesshaftigkeit der Genese von common ground beachtet werden, da im Verlauf einer Interaktion solcher common ground erarbeitet wird und Auswirkungen auf die Interaktionen hat. Während zu Beginn einer Interaktionsgeschichte beispielsweise noch ein großer verbaler Aufwand nötig ist, Handlungsbedeutung zu erstellen, reichen zu einem späteren Zeitpunkt Stichwörter oder Gesten, um Gleiches zu erreichen (vgl. Deppermann 2015, 344 für ein illustratives Beispiel einer Fahrstunde und dem Üben des Einparkens).

Ein Gesprächstranskript gibt in jedem Moment das wieder, was verbal - und je nach Transkriptionstiefe auch para- oder nonverbal - geäußert wird. Die Sedimente der Interaktionsgeschichte sind nicht sichtbar und müssen rekonstruiert werden. Denkbar wäre jedoch, diagrammatische Mittel zu finden, die wenigstens 
die Spuren des Vergangenen erhalten, damit die Rekonstruktion der Interaktionsgeschichte leichter wird.

Es steht außer Frage, dass viele gesprächsanalytische Studien mit einer Pragmatiktheorie, wie sie Deppermann skizziert, arbeiten. Die theoretischen Veränderungen der Gesprächslinguistik führten auch zu methodischen Veränderungen der Transkription, wenn man an die Integration von Audio- und Videodaten, die exakte Alignierung mit der Transkription etc. denkt. Mir scheint aber, dass die Integration solcher Aspekte weiterhin der diagrammatischen Grundfigur der Partitur folgt und daher die Grundprobleme nicht löst, nämlich in der Sequenzialität gewissermaßen gefangen zu sein. Um die Entwicklungen der Theorie auch transkriptionsmethodisch zu reflektieren, ist eine grundsätzliche Hinterfragung der diagrammatischen Grundfigur nötig, ohne natürlich die Vorteile zu verlieren. Dass das Gesprächstranskript in der Form des Partiturprinzips eine Innovation war, da es Simultaneität und Sequenzialität gesprochener Sprache erstmals analysierbar machte, ist unumstritten. Im nächsten Abschnitt möchte ich aber Vorschläge machen, wie diese Darstellungsform durch alternative Visualisierungen ergänzt werden könnte.

\subsection{Vorschläge für Visualisierungsformen}

Es ist unmöglich, mit einer Visualisierung gesprochener Sprache die im vorherigen Abschnitt elementaren Aspekte von Leiblichkeit, Zeitlichkeit, Sozialität und Epistemizität gleichermaßen zu berücksichtigen - bzw. die Daten so zu visualisieren, dass sie hinsichtlich der oben beschriebenen Aspekte lesbar werden. Ziel der (zeichnerischen) Skizzen und Überlegungen in diesem Abschnitt ist es, diagrammatische Formen vorzuschlagen, auf denen komplexere Visualisierungen und Analysemethoden aufgebaut werden könnten.

Eine Grundüberlegung dabei ist, dass eine Vereinigung eher quantitativserieller und qualitativ-ethnomethodologischer Analysemethoden wichtig ist, um den interaktionstheoretischen Überlegungen gerecht zu werden. Daher stelle ich zunächst Diagramme vor, welche diesen Brückenschlag versuchen, dabei aber auf dem klassischen Gesprächstranskript beruhen. Danach schlage ich ein Diagrammtypus der „Jahresringe“ vor, um eine neue Art von Transkription zu ermöglichen. 


\subsubsection{Korpuslinguistik und Gesprächsanalyse}

Mit Fokus auf die Sozialität von Gesprächen ist auffallend, dass es unterschiedliche Beteiligungsstrukturen gibt, die unterschiedliche Rollen in einer Interaktion widerspiegeln (vgl. Abschnitt 8.1). Beim klassischen Gesprächstranskript, insbesondere wenn es konsequent in Partiturschreibweise verfasst ist, sind solche Rollen prinzipiell sichtbar. Vereinfacht zu einer grafischen Form sind beispielsweise Typen denkbar, wie in Abbildung 102 aufgeführt. Unverkennbar steht dabei die diagrammatische Grundfigur der Partitur Pate, die Verschriftlichung der turns wird jedoch zu Gunsten einer grafischen Abbreviatur ersetzt. Der Gewinn der grafischen Abbreviatur liegt darin, die strukturellen Besonderheiten zu betonen und die Gesprächsinhalte in den Hintergrund zu stellen.
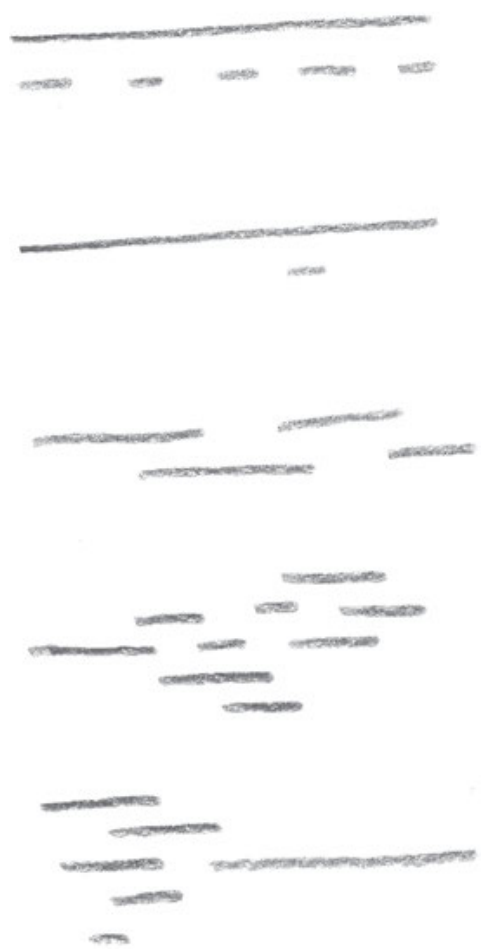

Abb. 102: Beteiligungsstrukturen grafisch visualisiert 
Wenn klassische Transkripte vorhanden sind, kann die grafische Umsetzung automatisiert aufgrund der turn-Struktur (wie sie beispielsweise bei in XML codierten Transkripten verfügbar sind) erstellt werden. Es handelt sich also um eine Sicht, bei der man aus weiterer Entfernung auf das Transkript schaut. So werden Strukturen sichtbar, wie Typen von Sprecherwechsel oder deren Häufigkeit und Dynamik, die als unterschiedliche „Beteiligungsstrukturen“ (Deppermann 2015, 338) aufgefasst werden können.

Während bei einem einzelnen Gesprächstranskript diese Sicht noch zu partikular ist, können bei einer größeren Datensammlung von Gesprächstranskripten wiederkehrende Strukturtypen gefunden werden, indem (automatisiert) ähnliche grafische Strukturen entdeckt werden. Damit kommt eine quantitative Sichtweise ins Spiel, mit der Einzelbeobachtungen in ihrer Serialität gewürdigt werden können.
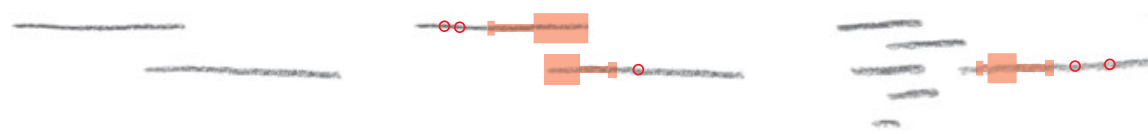

Abb. 103: Kombination von Typen und statistischen Angaben (Boxplot)

Wenn Sequenzen in den Transkriptdaten nach Beteiligungsstrukturen typologisiert werden können, ist es naheliegend, diese Typen mit bestimmten weiteren Eigenschaften korrelieren zu lassen. So könnte man sich bei einem bestimmten Typus von turn-taking dafür interessieren, welches lexikalische Material dort - und wo genau dort - typischerweise auftritt. Solche quantitativ erfassbaren Merkmale können dann statistisch ausgewertet und in einem kombinierten Diagramm dargestellt werden, das beispielsweise eine Boxplot- mit der Partiturdarstellung kombiniert (vgl. Abbildung 103). Der Boxplot zeigt dabei beispielsweise an, wo genau - wie viele Wörter vor oder nach dem Sprecherwechsel und bei welcher Stimme - Wortwiederholungen bei solchen Sprecherwechseln auftreten.

Nach dem gleichen Prinzip können Gesprächsdynamik-Typen grafisch abgekürzt werden. Abbildung 104 zeigt eine entsprechende Skizze: oben ist das Prinzip dargestellt, mit dem die einzelnen turns Knoten eines Graphen darstellen, so dass sich eine sequenziell ausgerichtete Graphdarstellung ergibt. Dieser Graph kann wiederum (automatisch) vereinfacht werden, so dass durch die Zickzack-Bewegung mit der Höhe der Zacken sowohl die Anzahl beteiligter Sprecher/ innen, als auch mit der Amplitude (der Distanz zwischen den Wendepunkten) die turn-Längen ausgedrückt werden können. 

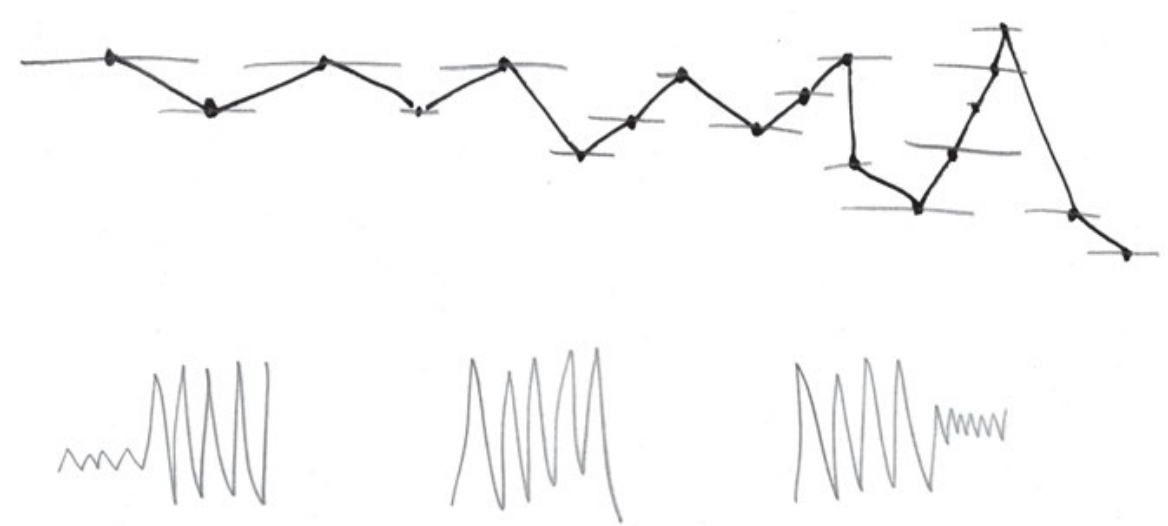

Abb. 104: Grafische Abbreviaturen von Gesprächsdynamik

Schließlich können die verschiedenen Darstellungsarten in Kombination benutzt werden. Ein mögliches Szenario ist in Abbildung 105 (s. u., S. 288) dargestellt: Wenn ein Korpus von Transkripten beispielsweise nach bestimmten Typen von Beteiligungsstrukturen klassifiziert ist, wäre eine Abfrage nach bestimmten Typen möglich. Man erhält dabei eine KWiC-Liste von Gesprächsausschnitten, die entsprechend klassifiziert worden sind, wenn gewünscht mit bestimmten Einschränkungen, z. B. mit der Einschränkung, dass im Bereich bestimmte Lexeme (im Beispiel: aber) vorkommen muss.

Eine alternative Darstellung der gleichen Sequenzen würde stärker die Gesprächsdynamik hervorheben, wie im zweiten Diagramm in Abbildung 105, das das Amplituden-Diagramm von Abbildung 104 wieder aufnimmt. Dieses Diagramm repräsentierte entweder eine einzelne Gesprächssequenz, oder aber auch viele weitere ähnliche Sequenzen in aggregierter Form. Als interaktives Diagramm umgesetzt, wäre es möglich, bestimmte Positionen innerhalb der typischen Sequenz auszuwählen und weitere Informationen dazu aufzurufen. So könnten beispielsweise die Transkriptausschnitte (oder Audio- und Videospuren) oder aber statistische Auswertungen, z. B. über die statistische Verteilung von verbalem, paraverbalem oder nonverbalem Material, aufgerufen werden. Das dritte Diagramm in Abbildung 105 ist ein einfaches Balkendiagramm, das anzeigt, wie oft aber an der markierten Position des Gesprächsdynamik-Typs auftritt - und wie oft an anderen Positionen. 


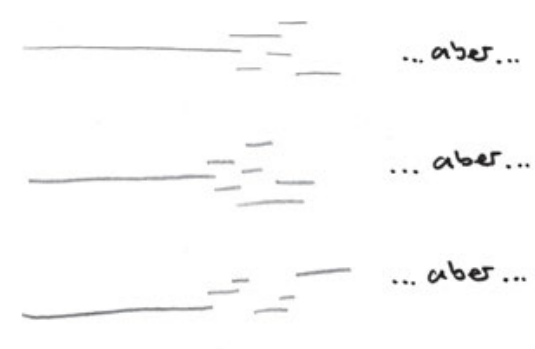

KWiC

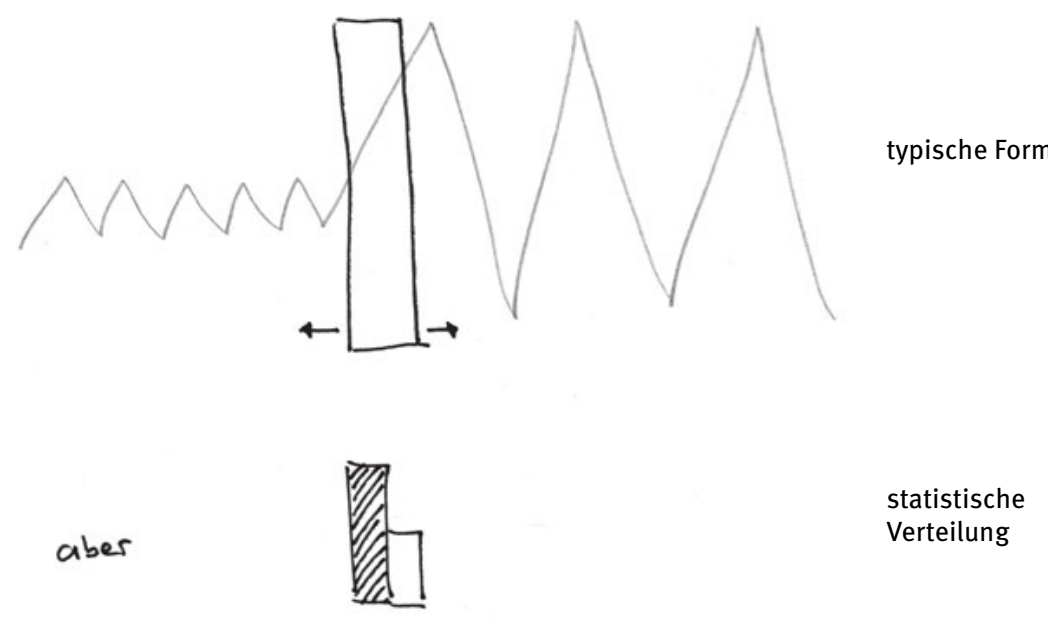

Abb. 105: Verschiedene Diagramme in Kombination

Die gemachten Vorschläge sind weder besonders innovativ noch ausgegoren, sondern illustrieren einen Denkansatz, mit dem eingeführte diagrammatische Grundfiguren in einer bestimmten Disziplin und in einem bestimmten Denkkollektiv hinterfragt werden können, wenn man versucht, dort nicht übliche diagrammatische Grundfiguren einzuführen. Die oben vorgeschlagenen Amplituden-Diagramme zur Visualisierung von Gesprächsdynamik benutzen die diagrammatische Grundfigur des Vektors, die in der Gesprächsanalyse ansonsten keine Verbreitung findet, denn das klassische Gesprächstranskript geht auf die diagrammatische Grundfigur der Partitur zurück. Eine in der Gesprächsanalyse bislang nicht häufig verwendete diagrammatische Grundfigur kann so produktiv gemacht werden, indem sie an die Analyseinteressen und Daten der Gesprächsanalyse angepasst wird.

Zusätzlich liegen mit der quantitativ-korpuslinguistischen Perspektive, die bei diesen Beispielen auf Gesprächsdaten eingenommen wird, wiederum andere diagrammatische Grundfiguren nahe (KWiC, Diagramme der deskriptiven Statis- 
tik). Damit konfrontiert ergeben sich neue Formen wie die grafischen Abbreviaturen von Beteiligungsstrukturen oder Gesprächsdynamiken, die sowohl deutlich gesprächslinguistisch geprägt sind (Partitur, Sequenz), als auch korpuslinguistisch (aggregierende Darstellung), woraus sich eine neue methodisch-methodologische Perspektivierung ableiten lässt.

\subsubsection{Jahresringe}

Die Skizzen im vorherigen Abschnitt basierten wesentlich auf der diagrammatischen Grundfigur der Partitur. Wenn die zeitliche Struktur einer Gesprächsinteraktion besser berücksichtigt werden soll, dann muss die Linearität der Partitur aufgebrochen werden. Zudem soll der Aspekt der Sozialität von Gesprächen besser repräsentiert werden können, indem ein „Gesprächsraum“ diagrammatisch dargestellt werden soll.

Die Grundidee des folgenden Vorschlages liegt darin, die Jahresringe eines Baumes als Metapher für Gespräche zu verwenden, um eine neue diagrammatische Form zu entwickeln. Jahresringe spiegeln eine Wachstumsgeschichte wider, ähnlich wie im Verlauf einer Gesprächsinteraktion die Äußerungen und Interaktionen eine Spur hinterlassen und eine gemeinsam konstruierte Handlungsbedeutung begründen.

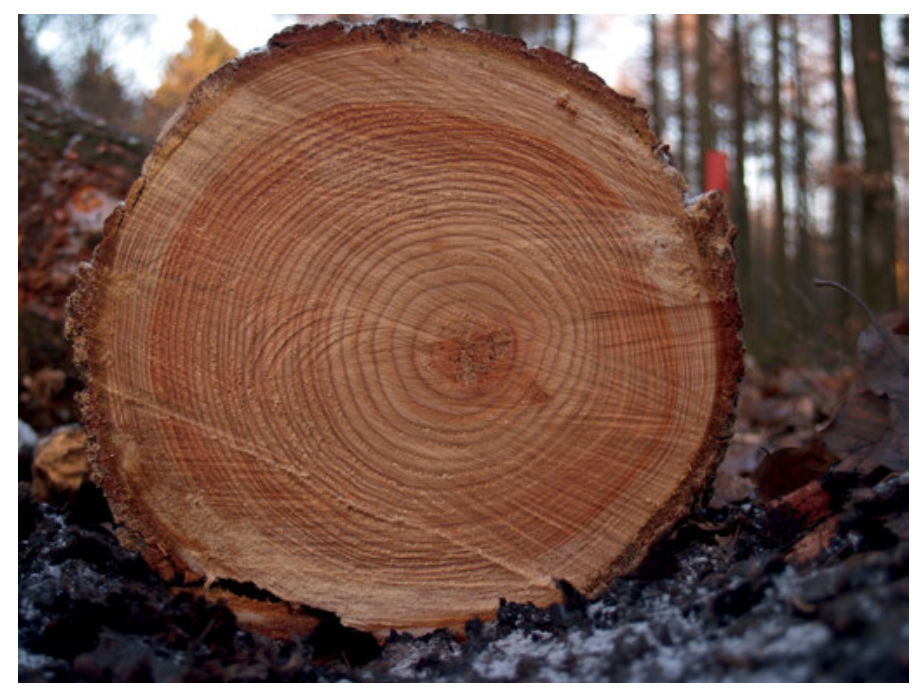

Abb. 106: Jahresringe als Metapher für Gespräche (Foto: Arnoldius, https://commons. wikimedia.org/wiki/File:Tree_rings.jpg, letzter Zugriff: 22.9. 2020) 
Ausgangspunkt der Überlegungen ist ein traditionelles Gesprächstranskript mit Audiosignal. Für die folgenden Beispiele verwendete ich drei Gespräche aus dem FOLK-Korpus (Schmidt 2016a). ${ }^{97}$ Die Arbeitsoberfläche der Anwendung ist folgendermaßen organisiert: Auf der linken Seite findet das Transkript Platz. Daneben wird der Gesprächsraum als Kreis dargestellt, auf dessen Linie alle Teilnehmenden des Gesprächs aufgeführt sind (vgl. Abbildung 107). Parallel zum Verlauf des Gesprächs werden nun die turns ebenfalls kreisförmig dargestellt: Zentrum des Kreises ist die Position der Sprecherin auf dem Gesprächsraum-Kreis. Die Größe des Kreises richtet sich nach der Länge des turns. Je kleiner der Radius, desto kürzer der turn. Die Farbe der Kreise codiert die Sprecherin oder den Sprecher. Mit jedem turn entsteht ein Kreis, in dessen Mitte der turn in Textform während der Abspieldauer des turns aufscheint. Gerade nach dem Auftreten des turn-Kreises erscheint er farblich gefüllt, die Füllung verschwindet jedoch nach ein paar Sekunden. Die Kontur des Kreises bleibt aber bestehen. ${ }^{98}$

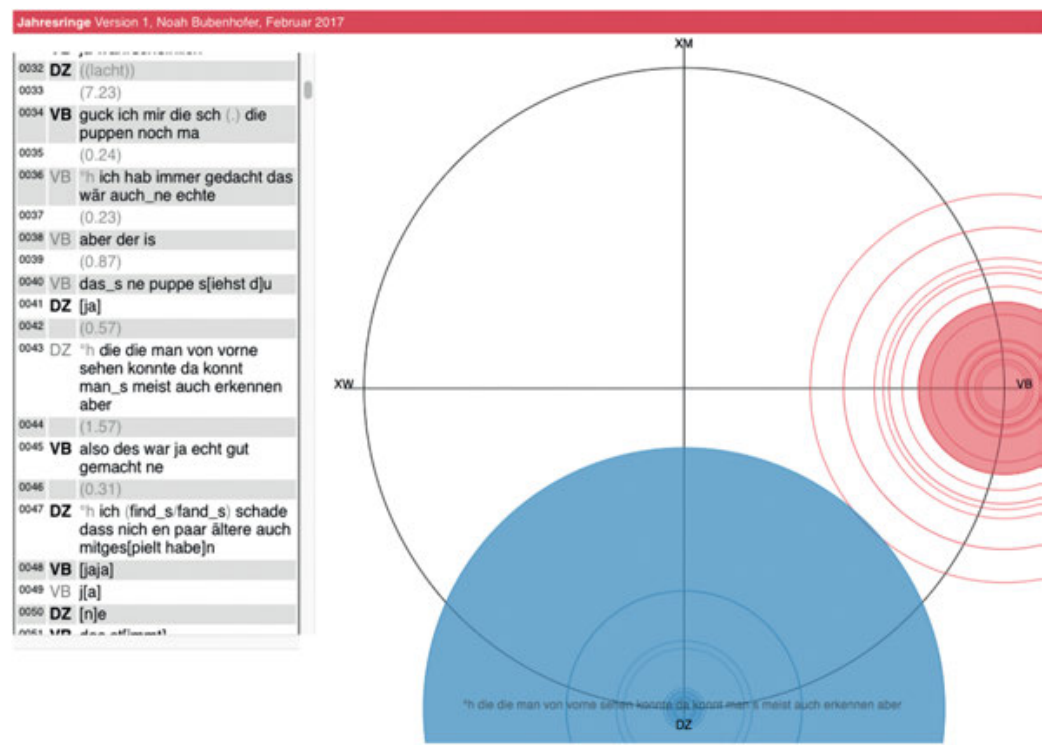

Abb. 107: Anwendung Jahresringe, Transkript und Jahresringe-Darstellung

97 Bei den Gesprächen handelt es sich um: FOLK_E_00080 - Alltagsgespräch: Pausenkommunikation im Theater; FOLK_E_00012 - Alltagsgespräch: Spielinteraktion mit Kindern; FOLK_E_00120 - Institutionelle Kommunikation: Unterrichtsstunde im Wirtschaftsgymnasium. 98 Auf der begleitenden Website www.bubenhofer.com/visuallinguistics/ kann die Anwendung online ausprobiert werden. 
Im Verlauf des Gesprächs ergeben sich somit Spuren, ähnlich Jahresringen eines Baumes, allerdings mit dem Unterschied, dass sich die Ringe nicht additiv umeinander anlegen und die Ringe immer größer werden. Stattdessen orientiert sich die Größe des Kreises alleine an der turn-Länge, so dass im Ergebnis die Reihenfolge der turns nicht abgelesen werden kann, sich jedoch ein Profil der turn-Frequenz und turn-Längen ergibt.

Mit dieser Form der Darstellung geht also die Sequenzialität des Gesprächs insofern verloren, als dass die Reihenfolge der turns keine Rolle mehr spielt (allerdings gibt diese das nebenstehende Transkript nach wie vor wieder). Jedoch wird der von Deppermann als elementar bezeichnete Aspekt der Zeitlichkeit des Gesprächs sichtbar, indem die Geschichte der verbalen Interaktion anhand des turn-Profils sichtbar wird. Dieser Mechanismus ist besonders dann interessant, wenn man Ergebnisse verschiedener Gespräche miteinander vergleicht (Abbildung 108). Das in der Mitte abgebildete Gespräch ist das gleiche wie in Abbildung 107. Obwohl es noch zwei weitere Akteure gibt, ist es geprägt durch den Dialog der beiden Sprecherinnen, die formal bezüglich turn-Längen ein ähnliches Gesprächsverhalten zeigen: Es findet sich die ganze Palette von kurzen und längeren turns mit einem Gewicht auf mittellangen turns.
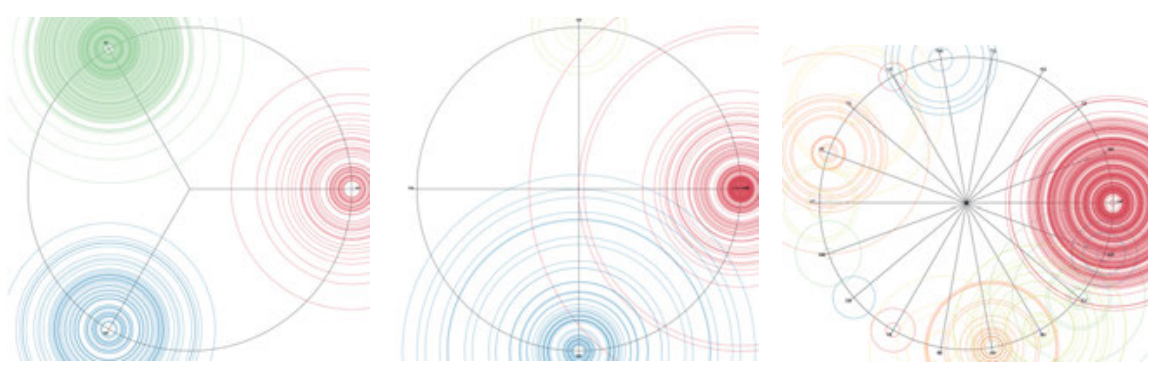

Abb. 108 : Drei Gespräche im Vergleich: Spielinteraktion mit Kindern, Pausenkommunikation zweier Besucherinnen im Theater, Unterrichtsstunde Gymnasium

Das Gespräch links in Abbildung 108 gibt eine Spielsequenz eines Vaters mit zwei Kindern wieder. Der Vater ist mit Position links oben (grün) dargestellt. Auch hier finden sich bei allen Beteiligten die ähnliche Palette von turn-Längen, auffallend ist aber der Vater, der besonders viele turns äußert, die zudem eher kurz sind. Die turn-Profile der Kinder ähneln sich und heben sich gleichzeitig deutlich vom Profil des Vaters ab.

Nochmals von anderem Charakter ist die dritte Darstellung ganz rechts in Abbildung 108. Es handelt sich um eine Interaktion in einer Schulklasse. 
Unschwer ist die Lehrerin mit den meisten Gesprächsbeiträgen erkennbar und es ist deutlich, wer unter den Schülerinnen und Schülern mehr oder weniger beteiligt ist.
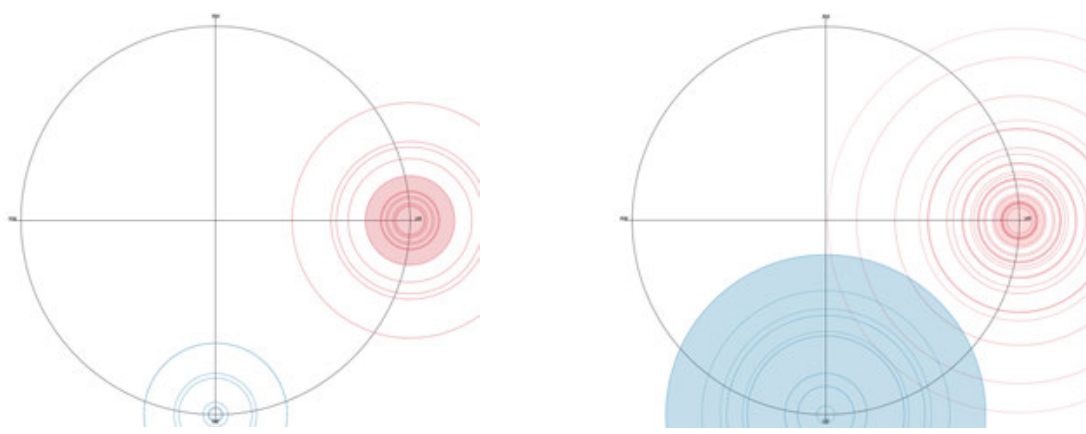

Abb. 109: Zwei verschiedene Zeitpunkte im gleichen Gespräch (Pausenkommunikation Theater)

Die Darstellung kann aber auch verwendet werden, um die turn-Profile zu verschiedenen Zeitpunkten im gleichen Gespräch zu analysieren (vgl. Abbildung 109). Das Beispiel zeigt wieder den Dialog zwischen den beiden Theaterbesucherinnen zu zwei unterschiedlichen Zeitpunkten. Beim zweiten Zeitpunkt (rechts) ist das Gespräch inzwischen geprägt von längeren turns, wo es um eine inhaltliche Diskussion geht, während beim früheren Zeitpunkt bislang noch eher organisatorische turns geäußert worden sind.

Technisch gesehen ist die Anwendung ,Jahresringe‘ in Javascript unter Verwendung der Javascript-Bibliothek „Paper.js“99 erstellt. Diese Bibliothek vereinfacht die dynamische Erstellung von Vektorgrafiken mit Javascript auf Basis der HTML5-Canvas-Technologie. Das Script liest automatisch das in HTML verfügbare Transkript aus und erstellt daraus das Diagramm.

Die Anwendung ist ein erstes Modell, das dazu dienen kann, die Eignung in Bezug auf die analytischen Bedürfnisse von Gesprächen zu testen. Viele weitere Ausbaustufen sind denkbar:

- Bessere Integration der verbalen Informationen, indem beispielsweise im Verlauf des Gesprächs wieder aufgenommene Themen oder Konzepte als Stichwörter im Gesprächsraum stehen bleiben. Dafür könnte mit korpuslinguistischen Mitteln zu jedem Zeitpunkt des Gesprächs berechnet werden,

99 Vgl. www.paperjs.org (letzter Zugriff: 22. 9. 2020). 
welche Wörter auffällig sind, weil sie beispielsweise neu auftauchen oder aber im Gespräch bleiben.

- Dynamischere Darstellung der Gesprächsdominanz, etwa indem bei wiederholter Gesprächsübernahme sich die ganze Anordnung so dreht, dass der/ die dominante Sprecher/in oben steht oder aber mehr Raum einnimmt.

- Daneben sind viele Erweiterungen des Benutzerinterfaces denkbar, etwa Interaktivität, um sich jederzeit die Inhalte der turns wieder anzeigen lassen zu können, ein Zeitstrahl, um beliebig vor- und zurückspulen zu können etc.

Wichtig ist hier jedoch der Punkt, dass diese Darstellung nicht das Transkript ersetzen, sondern ergänzen soll. Und noch wichtiger ist, dass die Visualisierung nicht losgelöst von bestimmten Forschungsinteressen entwickelt werden kann. Daher soll für den Moment auch bei dieser Skizze bleiben, die als Basis für weitergehende Entwicklungen dienen kann, die von bestimmten Forschungsinteressen ausgehen.

\subsection{Fazit und diagrammatische Verortung}

Ausgangspunkt der Überlegungen für die dritte Fallstudie war, dass die interaktionale Linguistik mit neueren Theorien an Grenzen stoßen müsste, wenn sie für die Analyse weiterhin die klassischen Formen der Transkription von Gesprächen nutzt. Gesprächstrankriptionen, auch in ihrer digitalen Variante oder angereichert um weitere Spuren zur Integration von Videoaufnahmen, nonverbalen Zeichen oder zur Bewegung im Raum, folgen der diagrammatischen Grundfigur der Partitur. Diese Form führt weder zu einer Rekontextualisierung noch zu einer Desequenzialisierung, reichert die Daten jedoch mit raumzeitlichen Informationen an.

Um aber insbesondere Aspekte von Zeitlichkeit und Sozialität (Deppermann 2015, 327) von Gesprächen besser analysierbar zu machen, ist es angebracht, über die Verwendung anderer diagrammatischer Grundfiguren nachzudenken. So ist es beispielsweise schwer, anhand eines klassischen Gesprächstranskriptes den Verlauf der vorherigen Interaktionen komplett im Blick zu haben, um die aktuelle Situation zu rekonstruieren. Auch um die Beteiligungsstruktur des gesamten Gesprächs, zumindest in komplexeren Fällen, überblicken zu können, bedarf es einer eingehenden und wiederholten Lektüre der Transkription. Daher muss die Verwendung der diagrammatischen Grundfigur Partitur, die den Transkripten zugrunde liegt, in Frage gestellt werden, um einen neuen Blick auf die Daten gewinnen zu können. Alternativen sind Grundfiguren, die Sequenzialität 
modellieren können, gleichzeitig aber eine Daten aggregierende Perspektive ermöglichen.

Die auf der Basis der Figur der Jahresringe entwickelte Darstellung versucht Sequenzialität mit dieser aggregierenden Perspektive zu verbinden. Zudem stellt sie eine Fläche dar, mit der ein sozial konstituierter Gesprächsraum repräsentiert wird. Dabei spielen drei diagrammatische Grundfiguren eine wichtige Rolle: Liste, Graph und Karte.

- Karte: Die Definition der Fläche und der Positionierung der Sprechenden auf dieser Fläche folgt einer nichtgeografischen Karte mit einer räumlichen Verortung der Akteure. Es handelt sich jedoch nicht um die Abbildung des realen Gesprächsraumes, sondern um den Entwurf einer Ähnlichkeit, mit der zwar die realen Akteure und ihre potenziellen Relationen untereinander abgebildet werden, allerdings durch die gleichmäßige Anordnung der Akteure auf einem Grundkreis in einem neutralen Relationensystem. Akteure, die besonders intensiv miteinander interagieren sind nicht näher zueinander gesetzt. Erst im Verlauf des Gespräches und dem damit parallel sich verändernden Diagramm vollzieht sich auf diagrammatischer Seite eine Gewichtung der Relationen zwischen den Akteuren. Der in Abbildung 108 gezeigte Vergleich dreier Gespräche zeigt dies: Mit der Auflistung der turns als Kreise um die Akteurspositionen herum ergibt sich eine Gewichtung der Positionen im Gesprächsraum.

- Liste: Die Auflistung der Kreise, die turns repräsentieren, folgt der diagrammatischen Grundfigur der Liste und ist das entscheidende Prinzip, mit dem die aggregierende Sicht auf die Daten ermöglicht wird. Als ob die turns als Text in einer Liste aufgelistet würden, wird auch hier eine Liste erstellt, die aber grafisch funktioniert, um deutbare Spuren im Gesprächsraum hinterlassen zu können. Das Ensemble der Kreise, als Liste gelesen, weist Parallelen zum Schnitt durch einen Baumstamm auf, in dem die Jahresringe die Geschichte des Baumwachstums darstellen.

- Graph: Schließlich nimmt die Visualisierung Anleihen an der diagrammatischen Grundfigur Graph, wenn die grafischen turn-Listen der Akteure als Knoten in einem Netz, angeordnet auf einem ordnenden Kreis, wahrgenommen werden. Dieser Aspekt ist bis jetzt kaum ausgebaut: Würde das Diagramm stärker als Graph aufgefasst, könnten die Knoten ihre Position verändern, etwa in Abhängigkeit der Veränderung der Beteiligungsstruktur.

Die im Abschnitt oben skizzierten Ausbaustufen zeigen, dass mit den „Jahresringen“ ein Visualisierungsprinzip gefunden ist, das einen weiten Möglichkeitsraum eröffnet. Es ist mit anderen Diagrammen kombinierbar und ausbaubar; insofern kann von einem reichen Visualisierungsprinzip gesprochen werden. 
Die Coding Culture, in der dieser Prototyp entstanden ist, ist ähnlich mit derjenigen, die bei den Geokollokationen und den Narrativen wirkte. Eine kleine Abweichung ist insofern feststellbar, als dass die dafür verwendete Javascript-Bibliothek „Paper.js“ eine andere Zielgruppe anspricht als etwa die D3.js-Bibliothek. Ein Blick auf die auf der Website der Bibliothek aufgeführten Beispiele ${ }^{100}$ macht dies evident, da es kaum darum geht, nur Daten zu visualisieren, sondern ein viel breiterer Anwendungsbereich ersichtlich wird: Grafik, interaktives Design und digitale Kunst. Mit einer solchen Bibliothek ist deutlich mehr Aufwand nötig als etwa mit D3.js, um eine kanonische Datenvisualisierung zu programmieren, dafür verleitet sie dazu, ganz andere Formen digitaler Grafik auszuprobieren.

Dieser Ansatz verstößt gegen bestimmte Prinzipien der Gesprächsanalyse und der Transkription. So wird in vielen Anleitungen zur Gesprächstranskription betont, dass eine detaillierte und genaue Transkription unumgänglich sei, so bereits Sacks et al., die eine Transkription auf einem ,appropriate level of detail and precision“ (Sacks et al. 1974, 734) fordern, oder Deppermann der betont, dass das „genaue Hinhören und die ,demütige“ Verschriftung von Details, so merkwürdig, widersinnig oder unwichtig sie auch zu sein scheinen“ (Deppermann 2008, 40), entscheidend sind.

Diese Forderung, grundsätzlich alle Aspekte eines Gesprächs zunächst für relevant $\mathrm{zu}$ halten und $\mathrm{zu}$ dokumentieren, ist zweifellos wichtig und war wissenschaftshistorisch innovativ. Gleichzeitig besteht die Gefahr, allzu sehr einem bestimmen Denkstil verhaftet zu sein, mit dem andere Perspektiven auf gesprochene Sprache, stärker quantitative, abstrahierende und generalisierende, nicht auf der Hand liegen. Ein diagrammatischer Blick und das Experiment mit unorthodoxen diagrammatischen Grundfiguren, im vollen Bewusstsein der dadurch ausgelösten Veränderungen bei der Gegenstandskonstitution, mag ein Weg sein, neue Innovationen auszulösen.

100 Vgl. http://paperjs.org/examples/ (letzter Zugriff: 22. 9. 2020). 
